Tropical Journal of Pharmaceutical Research September 2015; 14 (9): 1679-1684

ISSN: 1596-5996 (print); 1596-9827 (electronic)

(C) Pharmacotherapy Group, Faculty of Pharmacy, University of Benin, Benin City, 300001 Nigeria.

All rights reserved.

Available online at http://www.tjpr.org

http://dx.doi.org/10.4314/tjpr.v14i9.19

Original Research Article

\title{
TaqMan Real-Time Polymerase Chain Reaction and Pyrosequencing using Single Nucleotide Polymorphism Protocol for Rapid Determination of ALDH2 *2 in a Chinese Population
}

\author{
Ju-yi Li ${ }^{1}$, Jin-hu Wu ${ }^{2}$, Yan Zhang ${ }^{2}$, Xiu-fang Wang ${ }^{2}$, Jie $\mathrm{Jin}^{2}$ and Yi Wang ${ }^{1 *}$ \\ ${ }^{1}$ Department of Pharmacy, The Central Hospital of Wuhan, ${ }^{2}$ The Third Hospital of Wuhan, Wuhan, Hubei Province, China
}

*For correspondence: Email: wxfls727@163.com

Received: 6 March 2015

Revised accepted: 26 July 2015

\begin{abstract}
Purpose: To establish a rapid molecular method for the detection of aldehyde dehydrogenase 2 (ALDH2), and determine whether the polymorphic ALDH2 gene is associated with drinking behavior in a Chinese population.

Methods: The gene polymorphism of ALDH2 *2 was detected using pyrosequencing and TaqMan realtime polymerase chain reaction (PCR) techniques. Genotyping of 176 volunteers were performed at The Third Hospital of Wuhan, Hubei, China. Genetic associations with alcohol use behavior was assessed. Results: Pyrosequencing and TaqMan real time PCR methods were successfully developed to identify ALDH2 *2 polymorphisms. The allele frequencies of ALDH2 *2 were $21.31 \%$ in the Chinese population. While the genotype frequency of heterozygous ALDH2 * $1 /{ }^{*} 2$ plus homozygous ALDH2 *2/ *2 was 46.67 $\%$ in non-drinkers, it was $30.17 \%$ in the alcoholics group $(p=0.030)$. The allele frequency of ALDH2 *2 was $15.09 \%$ in the alcoholics group and $28.33 \%$ among non-drinkers $(p=0.011)$. There were substantial differences in allele frequencies of ALDH2 genotypes between this study's Chinese sample and other ethnic groups in Asia; the frequency of ALDH2 *2 allele was significantly higher than that in European and Americans.

Conclusion: The developed pyrosequencing and TaqMan real time PCR methods are rapid, accurate, high-throughput, convenient, and reliable for detecting ALDH2 polymorphisms. ALDH2 *2 gene protects against the development of alcoholism. The frequencies of ALDH2 in this Chinese population differ from those of other ethnic populations.
\end{abstract}

Keywords: Aldehyde dehydrogenase 2, Polymorphism, TaqMan real time Polymerase Chain Reaction, Pyrosequencing, Allele, Alcoholics, Genotype frequency

Tropical Journal of Pharmaceutical Research is indexed by Science Citation Index (SciSearch), Scopus, International Pharmaceutical Abstract, Chemical Abstracts, Embase, Index Copernicus, EBSCO, African Index Medicus, JournalSeek, Journal Citation Reports/Science Edition, Directory of Open Access Journals (DOAJ), African Journal Online, Bioline International, Open-J-Gate and Pharmacy Abstracts

\section{INTRODUCTION}

Alcohol in humans is oxidized to acetaldehyde, which in turn is oxidized to harmless acetate by aldehyde dehydrogenases [1]. Aldehyde dehydrogenases-2 (ALDH2) is the major enzyme for acetaldehyde elimination, and its polymorphisms determine blood acetaldehyde concentrations after alcohol consumption. The Glu487Lys polymorphism (ALDH2 *2) has been the most frequently studied. Such a polymorphism ( $G$ to $A$ ) generates an ALDH2 with much lower activity and causes much higher blood levels of acetaldehyde, which may contribute to susceptibility to carcinogenesis [2]. The Glu487Lys polymorphism has been reported 
to be associated with many types of cancer, such as esophageal cancer [3]. For further analysis of these Single Nucleotide Polymorphisms (SNPs) and their relations to clinical outcomes, an accurate, rapid, and cost efficient method of SNP evaluation is needed, and it will have a positive roles to understand an individual's ALDH2 genetic condition which will improve the quality of life.

The conventional method for detection of the ALDH2 *2 mutation uses a PCR - RFLP (restriction fragment length polymorphism) followed by restriction enzyme digestion and agarose gel electrophoresis [4], which is both labor-intensive and time-consuming. Pyrosequencing was a DNA sequencing technique that was based on the detection of the pyrophosphate released during DNA synthesis [5], and was well suitable for large-scale screening for a short length DNA fragment. TaqMan genotyping assay with the real time PCR is a gold standard for the analysis of a limited number of SNPs in large sample collections, specifically when the site of an SNP is not detectable with a restriction enzyme. It allows the detection of both SNP alleles in a single reaction tube.

In this study, our aim was to establish a rapid molecular method for the detection of ALDH2 by pyrosequencing and TaqMan real time PCR technologies to investigate the gene polymorphism of ALDH2 *2 in a Chinese population, and comprehensive comparison with other populations.

\section{EXPERIMENTAL}

\section{Subjects and methods}

From December 2013 to June 2014, the participants, including hospital employers, general blue - collar laborers, and white-collar workers, who were excluded from the immunorelated diseases and other critical diseases, included 176 subjects (101 males and 75 females, with an average age of $39.86 \pm 9.45$ years) in this study. Each participant signed and completed questionnaires, including the data about his/her age, weight, height, education level, lifestyle, and self-reported alcohol intake status. All participants were requested to provide a blood sample. All study participants will sign an informed consent to agree to participate in the study in compliance with the Declaration of Helsinki [6]. This study was approved by the Ethics Committee of the Hospital of Wuhan (approval ref. no. 20131201). Three milliliters of fasting venous blood was collected from all subjects, and then the serum and blood clotting were separated. Genomic DNA was extracted from white blood cells using the TIANamp Blood DNA kit (Tiangen, beijing, China) following standard protocols.

\section{TaqMan real time PCR for ALDH*2 genotyping}

Primers and probes were synthesized by the $A B I$ Company (USA). A $25 \mu \mathrm{L}$ reaction mixture including $2 \mu \mathrm{L}$ DNA, $23 \mu \mathrm{L}$ TaqMan PCR master mix, primers, probes and deionized water (YZY Medical Science \& Technology Co., Ltd, Wuhan, China) was placed in an MX3000P real - time PCR instrument (Stratagene Corp, USA). The reaction conditions were as follows: initial treatment with uracil - $\mathrm{N}$ - glycosylase at $95{ }^{\circ} \mathrm{C}$ for $10 \mathrm{~min}$, denaturation at $95{ }^{\circ} \mathrm{C}$ for $10 \mathrm{~min}$, followed by 40 cycles of denaturation at $95^{\circ} \mathrm{C}$ for $15 \mathrm{~s}$, and annealing and extension at $60^{\circ} \mathrm{C}$ for $45 \mathrm{~s}$. The results were analyzed by the MX3000P Real Time PCR System, using the MxPro QPCR 4.10d Software (Agilent).

\section{Pyrosequencing for ALDH2 *2 genotyping}

DNA amplification: One of the known primers of ALDH2 *2 was used for amplification of DNA for PCR analysis was biotinylated, respectively. Primers for pyrosequencing of ALDH2 *2 was designed with PyroMark Assay Design Software 2.0. In the PCR assay (PyroMark PCR Kit Qiagen), we used a forward primer that was biotinylated at the $5^{\prime}$ - end of the ALDH2 ${ }^{*} 2$. The assay was performed in a $25 \mu \mathrm{L}$ reaction volume. The PCR conditions consisted of initial denaturation at $95{ }^{\circ} \mathrm{C}$ for $15 \mathrm{~min}$, followed by 45 cycles of denaturation at $94{ }^{\circ} \mathrm{C}$ for $30 \mathrm{~s}$, annealing at $60{ }^{\circ} \mathrm{C}$ for $30 \mathrm{~s}$, extension at $72{ }^{\circ} \mathrm{C}$ for $30 \mathrm{~s}$, and a final extension step at $72{ }^{\circ} \mathrm{C}$ for $10 \mathrm{~min}$. The PCR products were separated on 2 $\%$ agarose gels.

Biotinylated PCR products were immobilized on streptavidin - coated Sepharose beads (Streptavidin Sepharose High Performance, GE Healthcare). All of the streptavidin - coated Sepharose beads ( $2 \mu \mathrm{L}$ per sample) were mixed with binding buffer ( $40 \mu \mathrm{L}$ per sample) in a tube. High - purity water was then added to a total volume of $80 \mu \mathrm{L}$ per well, including the PCR product $(20 \mu \mathrm{L})$. This immobilization mix was incubated for $10 \mathrm{~min}$ at $25{ }^{\circ} \mathrm{C}$ with continuous mixing $(1400 \mathrm{rpm})$ on a shaking device, and the sequencing primer was then diluted to $0.3 \mu \mathrm{mol} / \mathrm{L}$ in annealing buffer. Next, $25 \mu \mathrm{L}$ of the solution was transferred to each well of a PyroMark Q24 Plate. 
After immobilization, the liquid was removed by aspirating the beads with a Vacuum Prep Tool and the beads were treated for approximately $5 \mathrm{~s}$ with $75 \%$ ethanol, $5 \mathrm{~s}$ with denaturation buffer, and $5 \mathrm{~s}$ with washing buffer. PyroMark Q24 Plate containing the samples was heated at $80^{\circ} \mathrm{C}$ for 2 min using a PyroMark Q24 Plate Holder and a heating block. The plate was then removed from the plate holder and the samples were allowed to cool to room temperature $\left(15-25^{\circ} \mathrm{C}\right)$ for at least $5 \mathrm{~min}$, and the reagents, including enzyme and substrate mixtures, and nucleotides were added to the cartridge (PyroMark Q24, Qiagen) [7]. The samples were analyzed using a PyroMark Q24 system (Qiagen) according to standard protocols. The order of nucleotide dispensation was chosen based on suggestions provided by PyroMark Assay Design Software 2.0.

\section{Statistical analysis}

Statistical analysis was performed using SPSS 11.5 software. One way ANOVA and t-test was used to compare mean differences for continuous variables. Allele frequency was determined via direct counting. The distribution of genotypes in all participants was obtained using Chi square test, and statistical significance was defined as $p<0.05$.

\section{RESULTS}

\section{Results of TaqMan PCR}

Two primers and one pair of fluorescenceconjugated probes were used in each reaction. The probe of allele $1(\mathrm{G})$, which matched with the wild type was labeled with FAM (color green); the probe of allele $2(A)$, which matched with the mutant was labeled with VIC (color red). Only significantly increased green fluorescence indicated the homozygous allele 1 (GG), only significantly increased red fluorescence indicated the homozygous allele $2(A A)$, and an increase in both colors of fluorescence signals indicated the heterozygous of allele 1 and allele 2 (AG).

\section{Pyrosequencing results}

We identified each SNP for ALDH2 *2 using a singlet pyrosequencing method. The assay was designed to generate a specific sequence for the SNP by setting a suitable nucleotide addition (Figure 1). The sequencing data obtained from the pyrosequencing method was validated by TaqMan PCR of the SNP for randomly selected samples, and the results showed $100 \%$ concordance with the TaqMan PCR results, indicating $100 \%$ specificity and sensitivity for the newly developed method.

\section{Genetic polymorphism of ALDH2 *2}

The frequency of the ALDH2 *2 allele was 21.31 $\%$, and that of the ALDH2 ${ }^{*} 1 /{ }^{*} 1,{ }^{*} 1 /{ }^{*} 2$ and ${ }^{*} 2 /$ *2 genotypes were $60.79,35.80$ and $3.41 \%$, respectively (Table 1 ). Table 1 show the genotype and allele frequencies of ALDH2 in each group. Whereas the genotype frequency of heterozygous ALDH2 *1/ *2 plus homozygous ALDH2 *2/ *2 was $46.67 \%$ in non-drinkers, they were $30.17 \%$ in the alcoholics group $(p=0.030)$. The allele frequencies of ALDH2*2 were $15.09 \%$ in the alcoholic group and $28.33 \%$ in nondrinkers $(p=0.011)$.

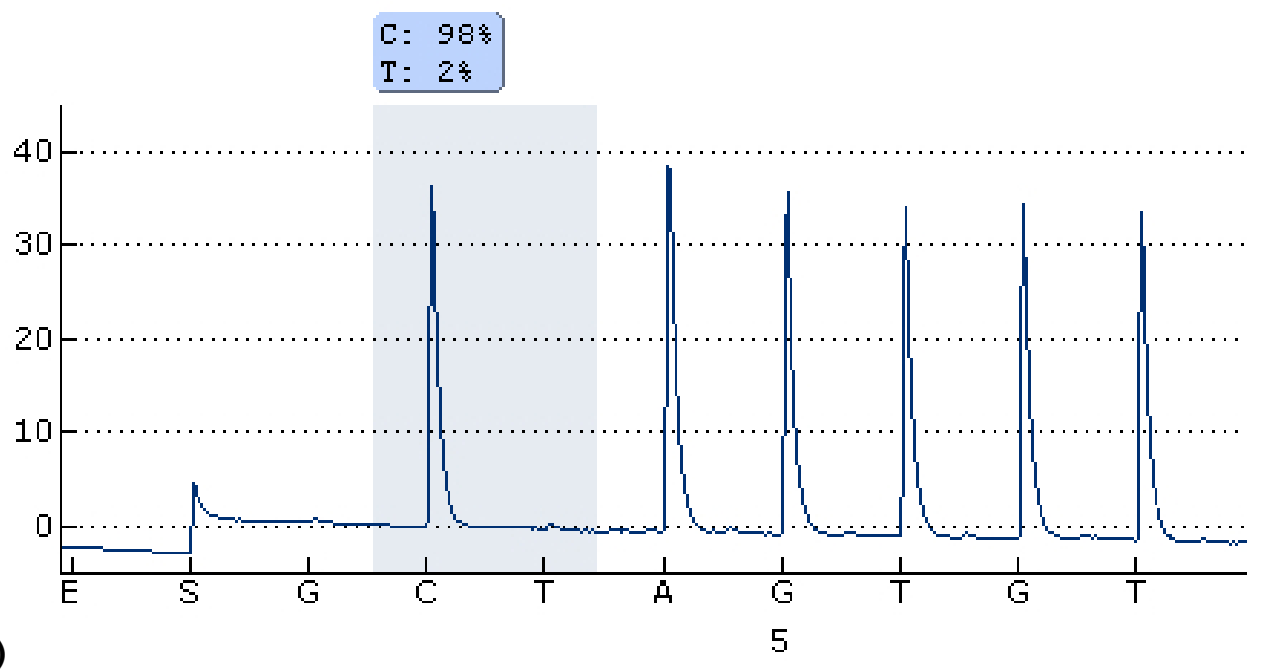

(A) 


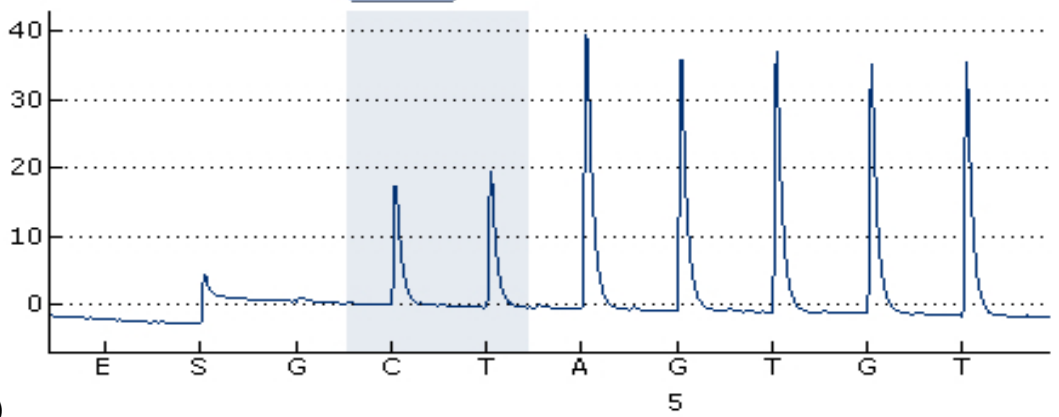

(B)

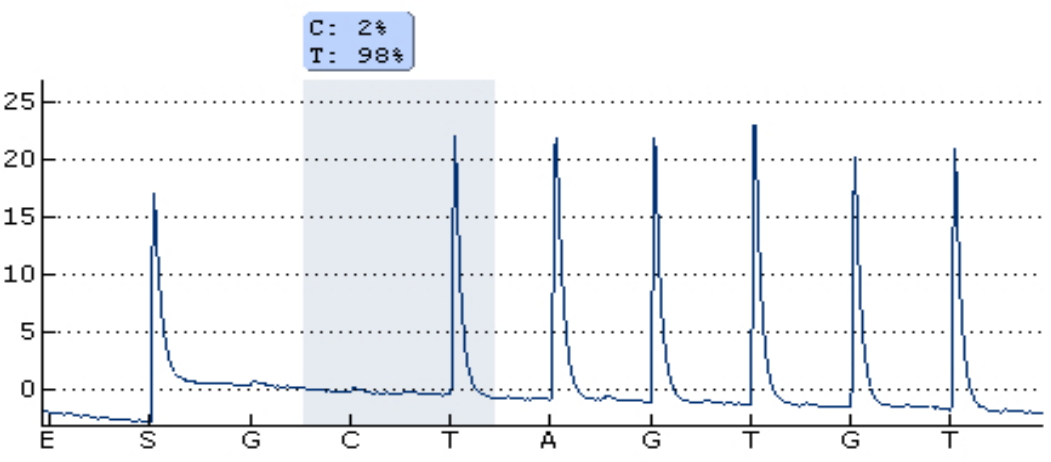

(C)

Figure 1: Pyrosequencing for ALDH2 *2 genotyping. The pyrograms for ALDH2 *2 in individuals presenting with the following genotypes: ALDH2 $* 1 /{ }^{*} 1$ (A), ALDH2 ${ }^{*} 1 /{ }^{*} 2$ (B), ALDH2 $* 2 /{ }^{*} 2(\mathrm{C})$. The results were detected by the method of reverse sequencing, thus, the detected CC genotype was the actual GG genotype. By the same inference, the detected CT / TT genotypes were the actual AG / AA genotype respectively

When we compared the allele frequencies of ALDH2 *2 polymorphisms in this population with those in other ethnic groups, the ALDH2 *2 polymorphism frequency were found to be similar to those reported for a Japanese population, but differed from those in other ethnic groups including Mexican Americans, Native Americans, Turkish, Spanish, Uzbekistan and Polish (Table 2).

Table 1: Genotype and allele frequencies of ALDH2

\begin{tabular}{lccccc}
\hline \multirow{2}{*}{ Group } & \multicolumn{3}{c}{ Genotype } & \multicolumn{2}{c}{ Allele frequency } \\
\cline { 2 - 6 } & AA (\%) & AG (\%) & GG (\%) & A (\%) & G (\%) \\
\hline Sample $(\mathrm{n}=176)$ & $107(60.79)$ & $63(35.80)$ & $6(3.41)$ & $75(21.31)$ & $277(78.69)$ \\
Alcoholic $(\mathrm{n}=116)$ & $0(0)$ & $35(30.17)^{*}$ & $81(69.83)$ & $35(15.09)^{* *}$ & $197(84.91)$ \\
Nondrinkers $(\mathrm{n}=60)$ & $6(10.00)$ & $22(36.67)$ & $32(53.33)$ & $34(28.33)$ & $97(71.67)$ \\
\hline${ }^{*} P=0.030$, compared with non-drinkers $($ AA plus GG), $" p=0.011$, compared with non-drinkers; percentage values \\
are in parenthesis
\end{tabular}

Table 2: Comparisons of ALDH2 *2 allele frequencies with those in other ethnic groups

\begin{tabular}{llcc}
\hline & Population & Frequency (\%) & Reference \\
\cline { 2 - 4 } & Japan & 22.30 & {$[8]$} \\
& Mexican Americans & 0.60 & {$[9]$} \\
& Native Americans & 0 & {$[9]$} \\
& Turkish & 0 & {$[10]$} \\
& Spanish & 0 & {$[11]$} \\
& Uzbekistan & 1.60 & {$[12]$} \\
& Korean & 16.70 & {$[13]$} \\
& Indian & 18.68 & {$[14]$} \\
& Polish & 2.48 & {$[15]$} \\
\hline
\end{tabular}

In Asia, the frequency of the ALDH2 *2 allele was significantly higher than in European and American populations 


\section{DISCUSSION}

In this study, we developed a rapid and robust pyrosequencing and TaqMan PCR methods to detect ALDH2 SNP and applied this technique to identify the SNP in a Chinese population, and we compared both procedures and found the results were the same. To our knowledge, this is the first study to identify ALDH2 polymorphisms using pyrosequencing and TaqMan PCR methods simultaneously. Previously, ALDH2 SNPs were detected through PCR-RFLP [4]. The pyrosequencing method presented here is directly applicable to studies involving genetic determination of ALDH2 polymorphisms in a large sample population.

A key advantage of pyrosequencing is that direct sequence data for each DNA are obtained which means that little interpretation is necessary compared with other methods, such as RFLP analysis, where incomplete digestion may complicate data analysis. Furthermore, the time and cost per sample for pyrosequencing are less than for RFLP analysis [16]. A method using realtime PCR offers an advantage over RFLP in that it can be modified for high-throughput applications, but it does not provide direct sequencing of DNA and therefore still requires some data interpretation. Compared with pyrosequencing, the real - time PCR method has less experimental procedures, the result of the genotype could be read directly by the outcome of PCR. Thus, pyrosequencing and TaqMan PCR methods have different strengths, they all showed high accurate, high-throughput, sensitivity, convenience.

ALDH2, located on chromosome 12q24.2, is the primary enzyme responsible for acetaldehyde metabolism in the liver, and thus, represents a logical candidate gene for alcohol dependence. A mutation in ALDH2 produces a largely inactive aldehyde dehydrogenase enzyme that leads to elevated acetaldehyde levels that produces an aversive flushing reaction when alcohol is consumed. This ALDH2 *2 allele is relatively frequent in Far East Asian populations and has been associated with lower rates of alcohol use and alcoholism in Japanese and Chinese samples [17].

In the present study, the frequency of the ALDH2 *2 allele was $22.31 \%$. We observed that there were substantial differences in allele frequencies of ALDH2 genotypes between our Chinese sample and other ethnic groups in Asian. The frequency of the ALDH2 *2 allele were significantly higher than European and America. The genotype frequency of heterozygous ALDH2
*1/ *2 plus homozygous ALDH2 *2/ *2 was 46.67 $\%$ in nondrinkers, they were $30.17 \%$ in the alcoholics group $(p=0.030)$. The allele frequencies of ALDH2 ${ }^{*} 2$ were $15.09 \%$ in the alcoholic group and $28.33 \%$ in nondrinkers $(p=$ $0.011)$, showing that ALDH2 *2 gene protected against the development of alcoholism, because of the unpleasant symptoms (facial flushing, tachycardia, nausea,) after drinking.

\section{Limitations of the study}

While the present study has provided much useful information on a rapid molecular method for the detection of ALDH2 and whether the polymorphic ALDH2 gene is associated with drinking behavior in a Chinese population, it has several limitations that must be acknowledged. In this study, the sample size of participants was small, the result of which may be bias, and hence a larger-sample study needs to be undertaken.

\section{CONCLUSION}

The developed pyrosequencing and TaqMan PCR methods are rapid and reliable genotyping methods to detect ALDH2 polymorphisms. ALDH2 *2 gene protects against alcoholism. There are large differences in the polymorphism of ALDH2 between the Chinese population in this study and those other ethnic groups.

\section{ACKNOWLEDGEMENT}

This work was supported by grants from Natural Science Foundation of Hubei Province, China (no. 2014CFC1043).

\section{REFERENCES}

1. Erikson CJ. The role of acetaldehyde in the actions of alcohol (update 2000). Alcohol Clin Exp Res. 2001; 25: S15-32.

2. Li H, Borinskaya S, Yoshimura K, Kal'ina $N$, Marusin A, Stepanov VA, Qin Z, Khaliq S, Lee MY, Yang Y, et al. Refined geographic distribution of the oriental ALDH2*504Lys (nee 487Lys) variant. Ann Hum Genet. 2009; 73: 335-345.

3. Zhang $G H$, Mai RQ, Huang B. Meta-analysis of $A D H 1 B$ and ALDH2 polymorphisms and esophageal cancer risk in China. World J Gastroenterol. 2010; 16: 60206025.

4. Ishikawa H, Miyatsu Y, Kurihara K, Yokoyama K. Geneenvironmental interactions between alcohol-drinking behavior and ALDH2 and CYP2E1 polymorphisms and their impact on micronuclei frequency in human lymphocytes. Mutat Res. 2006; 594: 1-9. 
5. Nyren P: The history of pyrosequencing. Methods $\mathrm{Mol}$ Biol. 2007; 373: 1-14.

6. World Medical Association. World Medical Association Declaration of Helsinki: Ethical Principles for Medical Research Involving Human Subjects. Seoul: From the 59th World Medical Association Assembly, 2008. http://www.wma.net/en/30publications/10policies/b3/1 7c.pdf.

7. Chiu KW, Nakano T, Chen KD, Hsu LW, Lai CY, Chiu HC, Huang CY, Cheng YF, Goto S, Chen CL, et al. Homogeneous phenomenon of the graft when using different genotype characteristic of recipients/donors in living donor liver transplantation. World $J$ Hepatol. 2013; 5: 642-648.

8. Morita K, Saruwatari J, Miyagawa H, Uchiyashiki Y, Oniki K, Sakata M, Kajiwara A, Yoshida A, Jinnouchi $H$, Nakagawa $K$, et al. Association between aldehyde dehydrogenase 2 polymorphisms and the incidence of diabetic retinopathy among Japanese subjects with type 2 diabetes mellitus. Cardiovasc Diabetol. 2013; 12: 132.

9. Konishi T, Luo HR, Calvillo M, Mayo MS, Lin KM, Wan YJ. $A D H 1 B^{*} 1, A D H 1 C^{*} 2$, DRD2 (-141C Ins), and 5HTTLPR are associated with alcoholism in Mexican American men living in Los Angeles. Alcohol Clin Exp Res. 2004; 28: 1145-1152.

10. Kayaaltı Z1, Söylemezoğlu T. Distribution of $A D H 1 B$, ALDH2, CYP2E1 ${ }^{*} 6$, and CYP2E1 ${ }^{*} 7 B$ genotypes in Turkish population. Alcohol. 2010; 44: 415-423.

11. Vidal F, Lorenzo A, Auguet $T$, Olona M, Broch $M$, Gutiérrez C, Aguilar C, Estupiñà $P$, Santos M, Richart
C, et al. Genetic polymorphisms of $A D H 2, A D H 3$, CYP4502E1 Dra-I and Pst-I, and ALDH2 in Spanish men: lack of association with alcoholism and alcoholic liver disease. J Hepatol. 2004; 41: 744-750.

12. Ahn KS, Abdiev S, Rahimov B, Malikov Y, Bahramov S, Okada $R$, Naito $M$, Hamajima $N$. Alcohol dehydrogenase $1 B$ and Aldehyde dehydrogenase 2 Polymorphisms in Uzbekistan. Asian Pac J Cancer Prev. 2009; 10: 17-20.

13. Ji YB, Tae K, Ahn TH, Lee SH, Kim KR, Park CW, Park $B L$, Shin $H D$. ADH1B and ALDH2 polymorphisms and their associations with increased risk of squamous cell carcinoma of the head and neck in the Korean population. Oral Oncol. 2011; 47: 583-587.

14. Vaswani M, Prasad P, Kapur S. Association of ADH1B and ALDH2 gene polymorphisms with alcohol dependence: a pilot study from India. Hum Genomics. 2009; 3: 213-220.

15. Cichoz-Lach H, Celiński K, Słomka M. Alcoholmetabolizing enzyme gene polymorphisms and alcohol chronic pancreatitis among Polish individuals. HPB (Oxford). 2008; 10: 138-143.

16. Aquilante CL, Lobmeyer MT, Langaee TY, Johnson JA. Comparison of cytochrome P450 2 C9 genotyping methods and implications for the clinical laboratory. Pharmacotherapy. 2004; 24: 720-726.

17. Ehlers CL, Liang $T$, Gizer IR. $A D H$ and $A L D H$ polymorphisms and alcohol dependence in Mexican and Native Americans. Am J Drug Alcohol Abuse. 2012; 38: 389-394. 